\title{
POLIMERIZAÇÃO DO METACRILATO DE METILA POR DISPERSÃO EM DIÓXIDO DE CARBONO SUPERCRÍTICO UTILIZANDO ESTABILIZANTE PDMS VINIL TERMINAL
}

\author{
P. F. OLIVEIRA ${ }^{1}$, E. D. ACOSTA ${ }^{1}$, R. A. F. MACHADO ${ }^{1}$ e D. BARTH ${ }^{2}$ \\ ${ }^{1}$ Universidade Federal de Santa Catarina, Departamento de Engenharia Química e Engenharia de \\ Alimentos, Brasil \\ ${ }^{2}$ Université de Lorraine, Laboratoire Réactions et Génie des Procedés (LRGP), UMR 7274, França \\ E-mail para contato: patricia@enq.ufsc.br
}

\begin{abstract}
RESUMO - O PMMA possui aplicações nas áreas médica, farmacêutica e de engenharia. Diferentes processos podem ser empregados na sua produção. Nesse trabalho, foi realizada a polimerização do MMA por dispersão em $\mathrm{CO}_{2}$ supercrítico. $\mathrm{O} \mathrm{CO}_{2}$ é inerte, facilmente recuperado e separado do produto. $\mathrm{O}$ monômero é solúvel em $\mathrm{CO}_{2}$ denso, diferentemente do PMMA, necessitando de um estabilizante para manter a dispersão. Compostos fluorados e polímeros à base de silicone são normalmente utilizados. O PDMS vinil terminal foi usado como estabilizante de reação nesse estudo. AIBN foi utilizado como iniciador. As reações foram conduzidas a $16 \mathrm{MPa} / 80{ }^{\circ} \mathrm{C}$ por $4 \mathrm{~h}$. A caracterização do produto foi realizada por MEV para avaliar a morfologia e estimativa do tamanho de partículas, SEC para determinação da massa molar e da polidispersão, $\mathrm{RMN}-\mathrm{H}^{+}$para verificação da reação e gravimetria para determinação de monômero residual. Os resultados confirmaram a adequação do processo e do estabilizante na produção das partículas de PMMA.
\end{abstract}

\section{INTRODUÇÃO}

O poli(metacrilato de metila), PMMA, pode ser obtido via polimerização em massa, solução, emulsão, suspensão ou dispersão, dependendo da aplicação final. À exceção da polimerização em massa e por dispersão, todas as demais técnicas utilizam água ou solventes orgânicos no processo.

A tecnologia baseada em fluidos supercríticos tem sido amplamente proposta para a produção de materiais nanoestruturados e polimerização de monômeros orgânicos. A utilização desse processo para produzir partículas muito finas é uma área de pesquisa relativamente recente, sendo uma das vantagens a estreita distribuição de tamanho das partículas obtidas, o que é de extrema importância na determinação da aplicação de polímeros (Reverchon et al., 2009; Park e Shim, 2003). À parte as vantagens apresentadas pela utilização de um solvente inerte, a polimerização por dispersão em $\mathrm{CO}_{2}$ supercrítico apresenta igualmente como fator positivo a obtenção de polímeros de alta pureza, visto que ao despressurizar o sistema, o solvente é eliminado e o produto resultante corresponde ao polímero puro. 
No fluido supercrítico, os polímeros são expandidos e plastificados, reduzindo assim a sua temperatura de transição vítrea. Dessa forma, a eliminação de monômero residual, a mistura de aditivos e a formação de espumas podem ser facilmente realizadas. Ainda, a cinética de polimerização é favorecida, visto que a difusão do monômero nas partículas poliméricas é beneficiada em função da plastificação (Park e Shim, 2003).

O processo de polimerização por dispersão se distingue do processo de polimerização em suspensão clássico, pois consiste, inicialmente, de um sistema homogêneo contendo monômero, solvente orgânico, iniciador solúvel na fase orgânica e agente estabilizante. O sistema torna-se heterogêneo no decorrer da reação, pois o polímero formado é insolúvel no solvente $\left(\mathrm{CO}_{2}\right.$ supercrítico). A polimerização ocorre na fase contínua, pela ligação entre monômero e radicais vivos, formados a partir da dissociação do iniciador, e pela ligação entre radicais de iniciador e cadeias poliméricas em crescimento, bem como entre as cadeias em crescimento.

Para a obtenção do PMMA por polimerização em meio disperso, é necessário estabilizar o meio reacional utilizando agentes estabilizantes. Polímeros e copolímeros fluorados, bem como polímeros à base de siloxanos são utilizados para estabilizar reações de polimerização de $\mathrm{MMA}$ em $\mathrm{CO}_{2}$ supercrítico (Han et al. 2009; Reverchon et al., 2009). De Simone et al. (1994) demonstraram que o poly(1,1-dehydroperfluorooctyl acrylate) estabiliza de maneira eficaz a polimerização do metacrilato de metila por dispersão em $\mathrm{CO}_{2}$ supercrítico.

Nesse trabalho, foi realizada a polimerização do poli(metacrilato de metila) (PMMA) em meio disperso, utilizando o dióxido de carbono $\left(\mathrm{CO}_{2}\right)$ em condições supercríticas. Para estabilizar a reação foi utilizado o poli(dimetil siloxano), vinil terminal. As condições experimentais tiveram como base o trabalho de Han et al. (2009), os quais realizaram a polimerização do metacrilato de metila em $\mathrm{CO}_{2}$ supercrítico utilizando diferentes condições operacionais e um agente de suspensão base siloxano. Diferentemente de outras pesquisas publicadas na área, nesse trabalho foi utilizado um estabilizante comercial base siloxano. Em sua maioria, os trabalhos publicados na literatura a respeito da polimerização em meio supercrítico fizeram uso de estabilizantes fluorados, de custo mais elevado do que os siloxanos, ou então, de copolímeros sintetizados. Nesse trabalho, ao utilizar um estabilizante base siloxano comercial pretende-se garantir um sistema sem variações devido à síntese de um reagente e, ainda, não onerar o processo com o uso de compostos fluorados.

De acordo com Han et al. (2009), o metacrilato de metila é solúvel em $\mathrm{CO}_{2}$ em condições superiores a $80{ }^{\circ} \mathrm{C}$ e $12 \mathrm{MPa}$. Tendo em vista que os melhores resultados para rendimento e qualidade do polímero obtido por Han et al. (2009) deram-se nas pressões avaliadas de 13.8 e $20.7 \mathrm{MPa}$, definiu-se como condição experimental a pressão intermediária de $16 \mathrm{MPa}$ e $80{ }^{\circ} \mathrm{C}$. Ainda de acordo com o estudo realizado pelos autores, acima dessa condição o baixo incremento no rendimento de produto, associado a pouca variação da massa molar e da sua distribuição, não compensa o aumento no consumo energético do sistema devido ao aumento da pressão do $\mathrm{CO}_{2}$.

A reação de polimerização do metacrilato de metila por dispersão em $\mathrm{CO}_{2}$ é influenciada pela solubilidade do monômero e das cadeias do polímero (PMMA) em crescimento. O PMMA não é solúvel em $\mathrm{CO}_{2}$ denso; no entanto o seu monômero apresenta solubilidade sob determinadas 
condições. Dessa forma, as propriedades do polímero formado estão relacionadas à pressão do sistema ao longo da reação. Como o metacrilato de metila é solúvel nas condições operacionais utilizadas nesse trabalho, o sistema metacrilato de metila (MMA)/ $\mathrm{CO}_{2}$ é homogêneo no início da reação de polimerização. À medida que a reação transcorre, as cadeias poliméricas formadas precipitam e o sistema $\mathrm{MMA} / \mathrm{CO}_{2} / \mathrm{PMMA}$ torna-se heterogêneo. $\mathrm{O}$ produto obtido nas reações conduzidas nesse trabalho está na forma particulada (microesferas de PMMA).

\section{MATERIAL E MÉTODOS}

\subsection{Reações de polimerização}

As reações de polimerização do metacrilato de metila em meio supercrítico foram conduzidas em um reator de aço inox encamisado, com volume interno de $100 \mathrm{~mL}$, diâmetro e altura internos de 40 e $80 \mathrm{~mm}$, respectivamente. O módulo de compressão consiste em um cilindro de $\mathrm{CO}_{2}$, banho termocriostático, bomba de membranas, trocador de calor e medidor de fluxo mássico. A temperatura de reação é medida com um termopar localizado no interior do reator. A dispersão é mantida por um módulo de agitação magnética, o qual possui uma haste com pás do tipo Rushton de $16 \mathrm{~mm}$ de diâmetro acopladas, situadas à $5 \mathrm{~mm}$ do fundo do reator. A velocidade de agitação foi fixada em 900 rpm. $\mathrm{O} \mathrm{CO}_{2}$ utilizado possui grau de pureza de 99,99 \%. O sistema reacional consiste, ainda, no monômero metacrilato de metila (grau de pureza $\geq 98.5 \%$; Fluka), no iniciador AIBN ( $\alpha, \alpha^{\prime}$-azoisobutironitrila; grau de pureza $98 \%$; Sigma-Aldrich) e no agente estabilizante poli(dimetil siloxano) vinil terminal (Sigma-Aldrich). A unidade experimental está ilustrada na Figura 1.

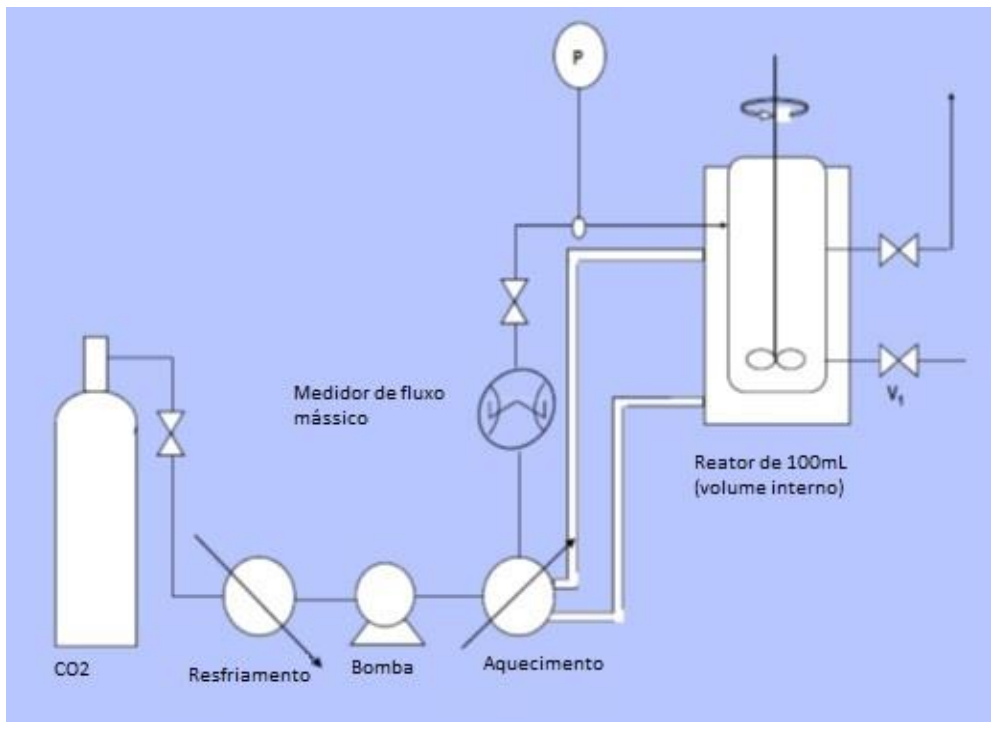

Figura 1 - Esquema da unidade experimental de polimerização à alta pressão.

Os reagentes (monômero, iniciador e estabilizante) são introduzidos no reator previamente aquecido a 35 oC. Uma vez que o reator é fechado, este é alimentado com CO2 de maneira gradual, simultaneamente com o aumento da temperatura, até que as condições da reação sejam atingidas. A alimentação de $\mathrm{CO} 2$ é interrompida uma vez que o sistema estabilize nas condições pretendidas. Após 
o término da reação $(4 \mathrm{~h})$, o sistema é resfriado e despressurizado à condição ambiente. O polímero, sob a forma de partículas finas é, então, recuperado, lavado com n-hexano para a retirada de resíduos do agente estabilizante, e filtrado à vácuo. A seguir, procede-se à secagem em estufa à 30 oC com circulação de ar. As condições experimentais utilizadas podem ser encontradas na Tabela 1.

Tabela 1 - Condições experimentais para a reação de polimerização do $\mathrm{MMA}$ em $\mathrm{CO}_{2}$ supercrítico

\begin{tabular}{|l|l|}
\hline Reação & $\mathrm{P}=16 \mathrm{MPa}$ \\
\hline & $\mathrm{T}=80^{\circ} \mathrm{C}$ \\
\hline & Agitação $=900 \mathrm{rpm}$ \\
\hline & Tempo de reação $=4 \mathrm{~h}$ \\
\hline Formulação & $\begin{array}{l}\text { Metacrilato de metila }=28.9 \% \mathrm{~m} / \mathrm{m} \text {, em relação à massa total do sistema; } \\
\rho=940 \mathrm{~g} / \mathrm{L}\left(80^{\circ} \mathrm{C}\right)\end{array}$ \\
\hline & AIBN $=0.48 \% \mathrm{~m} / \mathrm{m}$, em relação à massa de monômero \\
\hline & Estabilizante $=5.4 \%$ e $7.02 \% \mathrm{~m} / \mathrm{m}$, em relação à massa de monômero \\
\hline & $\rho_{\mathrm{CO} 2}=470 \mathrm{~g} / \mathrm{L}\left(16 \mathrm{MPa} / 80^{\circ} \mathrm{C}\right)$ \\
\hline
\end{tabular}

\subsection{Caracterização do polímero obtido}

O polímero obtido foi caracterizado quanto à massa molar, presença de monômero residual, morfologia e distribuição do tamanho de partículas, bem como a verificação das reações ocorridas durante o processo pela identificação dos compostos formados. Para isso, as amostras foram submetidas à análise por cromatografia de exclusão por tamanho (SEC, Size Exclusion Chromathography), gravimetria, microscopia eletrônica de varredura (MEV) e RMN-H , respectivamente. As análises foram realizadas na Université de Lorraine (LRGP, França) e na Universidade Federal de Santa Catarina (LCP/EQA).

\section{RESULTADOS E DISCUSSÃO}

Neste item são apresentados os resultados obtidos para as reações de polimerização do metacrilato de metila por dispersão em dióxido de carbono supercrítico, bem como a caracterização do polímero obtido.

A Tabela 2 apresenta os resultados obtidos para as reações de polimerização em dispersão do MMA em $\mathrm{CO}_{2}$ supercrítico. Os resultados obtidos para a massa molar e a sua distribuição estão em concordância com os dados publicados na literatura. Rosell et al. (2004) realizaram a polimerização do MMA por dispersão $\mathrm{CO}_{2}$ supercrítico, utilizando um estabilizante de reação fluorado. Os autores obtiveram um polímero com massa molar da ordem de $10^{5} \mathrm{~g} / \mathrm{gmol}$, utilizando parâmetros operacionais similares àqueles utilizados nesse trabalho. Han et al. (2009) obtiveram PMMA com massa molar da ordem de $10^{4} \mathrm{~g} / \mathrm{gmol}$, usando poli(dimetil siloxano)-metacrilato de glicidila conjugados como surfactante.

Tabela 2 - Reações de polimerização por dispersão do metacrilato de metila em $\mathrm{CO}_{2}$ supercrítico. 
Parâmetros operacionais, polímero recuperado, massa molar e índice de polidispersão.

\begin{tabular}{|c|c|c|c|c|c|c|}
\hline Reação & \% estabilizante & $\begin{array}{c}\text { Massa } \\
\text { polímero } \\
\text { recuperada }(\mathrm{g})\end{array}$ & $\begin{array}{c}\text { \% polímero } \\
\text { recuperado }\end{array}$ & $\begin{array}{c}\mathrm{M}_{\mathrm{n} \times} 10^{5} \\
(\mathrm{~g} / \mathrm{gmol})\end{array}$ & $\begin{array}{c}\mathrm{M}_{\mathrm{w} \times} 10^{5} \\
\mathrm{~g} / \mathrm{gmol})\end{array}$ & $\begin{array}{c}\text { PDI } \\
\left(\mathrm{M}_{\mathrm{w}} / \mathrm{M}_{\mathrm{n}}\right)\end{array}$ \\
\hline 1 & 5,4 & 1,48 & 8,7 & 1,465 & 5,279 & $\begin{array}{c}3,603 \pm \\
0,047\end{array}$ \\
\hline 5 & 5,4 & 8,46 & 49,6 & 2,468 & 4,866 & $\begin{array}{c}1,972 \pm \\
0,022\end{array}$ \\
\hline 7 & 5,4 & 8,21 & 50,3 & 1,562 & 5,317 & $\begin{array}{c}3,405 \pm \\
0,025\end{array}$ \\
\hline 8 & 5,4 & 8,09 & 48,2 & 1,222 & 3,900 & $\begin{array}{c}3,192 \pm \\
0,295\end{array}$ \\
\hline 9 & 5,4 & 9,66 & 59,0 & 1,154 & 2,368 & $\begin{array}{c}2,052 \pm \\
0,022\end{array}$ \\
\hline 10 & 5,4 & 9,31 & 56,2 & 2,409 & 4,843 & $\begin{array}{c}2,011 \pm \\
0,026\end{array}$ \\
\hline 11 & 7,02 & 8,16 & 49,9 & 1,135 & 2,314 & $\begin{array}{c}2,038 \pm \\
0,043\end{array}$ \\
\hline
\end{tabular}

A variação da massa molar do polímero é função do tipo e concentração de iniciador, da ocorrência de reações secundárias entre os compostos reagentes do sistema, da concentração de monômero, da interação entre as fases contínua e dispersa, entre outros fatores. $\mathrm{O}$ fato do $\mathrm{CO}_{2}$ ser inerte apresenta vantagens devido à ausência de transferência de cadeia para o solvente (Beuermann e Buback, 2005). Segundo Lacroix-Desmazes (2006), a polimerização por dispersão favorece a formação de partículas estáveis em meio supercrítico e a obtenção de massas molares e conversões elevadas. No entanto, reações secundárias podem ocorrer entre os compostos presentes no meio reacional. Os resultados obtidos mostram uma constância nos valores da massa molar do PMMA e da sua distribuição, indicada pelo índice de polidispersão (PDI).

Na Tabela 3 estão apresentados os resultados para monômero residual no produto final e o diâmetro médio de partículas. A conversão máxima obtida foi de, aproximadamente, $95 \%$, em massa. Rosell et al. (2004) encontraram valores também elevados para a conversão de PMMA (máximo de 98\%). O diâmetro de partículas do PMMA foi determinado a partir da análise das micrografias obtidas por MEV com o programa SizeMeter 1.1. Os resultados possuem variação não negligenciável entre si. Isso pode ser justificado pelas oscilações na pressão e temperatura do sistema ao longo das reações, sendo que o meio reacional é bastante sensível a quaisquer oscilações nos parâmetros operacionais, influenciando no produto final. 


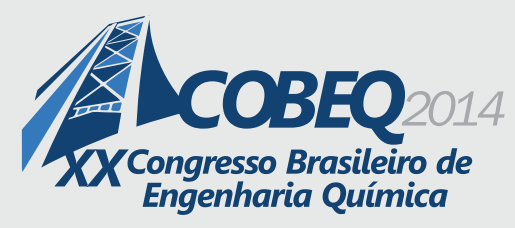

19 a 22 de outubro de 2014

Florianópolis/SC

Tabela 3 - Resultados para monômero residual e diâmetro médio de partículas. Diâmetro médio obtido utilizando o programa SizeMeter 1.1

\begin{tabular}{|c|c|c|c|c|}
\hline Reação & $\begin{array}{c}\text { Monômero residual } \\
(\%)\end{array}$ & Desvio padrão (\%) & $\begin{array}{c}\text { Diâmetro médio } \\
\text { das partículas }(\mu \mathrm{m})\end{array}$ & $\begin{array}{c}\text { Desvio padrão } \\
(\mu \mathrm{m})\end{array}$ \\
\hline 1 & 5,2 & 0,2 & 60,57 & 11,70 \\
\hline 5 & 5,2 & 0,5 & 29,67 & 8,50 \\
\hline 8 & 5,3 & 0,4 & 30,16 & 12,57 \\
\hline 9 & 5,5 & 0,1 & 19,86 & 8,29 \\
\hline 11 & 5,3 & 0,2 & 29,63 & 10,53 \\
\hline
\end{tabular}

$\mathrm{Na}$ Figura 2 são apresentadas as micrografias do PMMA obtido em $\mathrm{CO}_{2}$ supercrítico, demonstrando a forma esférica das partículas obtidas, coerente com os resultados da literatura (Lacroix-Desmazes, 2006; Beuermann e Buback, 2005; Rosell et al., 2004; De Simone et al., 1994; Han et al. 2009). Segundo Wang e Cheung (2004), a concentração de estabilizante controla a morfologia do polímero. Concentrações elevadas de estabilizante conduzem a tamanhos de partículas menores no sistema por dispersão em meio supercrítico. A reação 11 corresponde a um aumento de $30 \%$ na concentração de estabilizante (PDMS-vinil terminal). No entanto, o efeito do decréscimo no tamanho das partículas devido ao aumento da concentração de estabilizante não foi maior do que a instabilidade das condições operacionais, o que também causou uma variação nesse resultado.
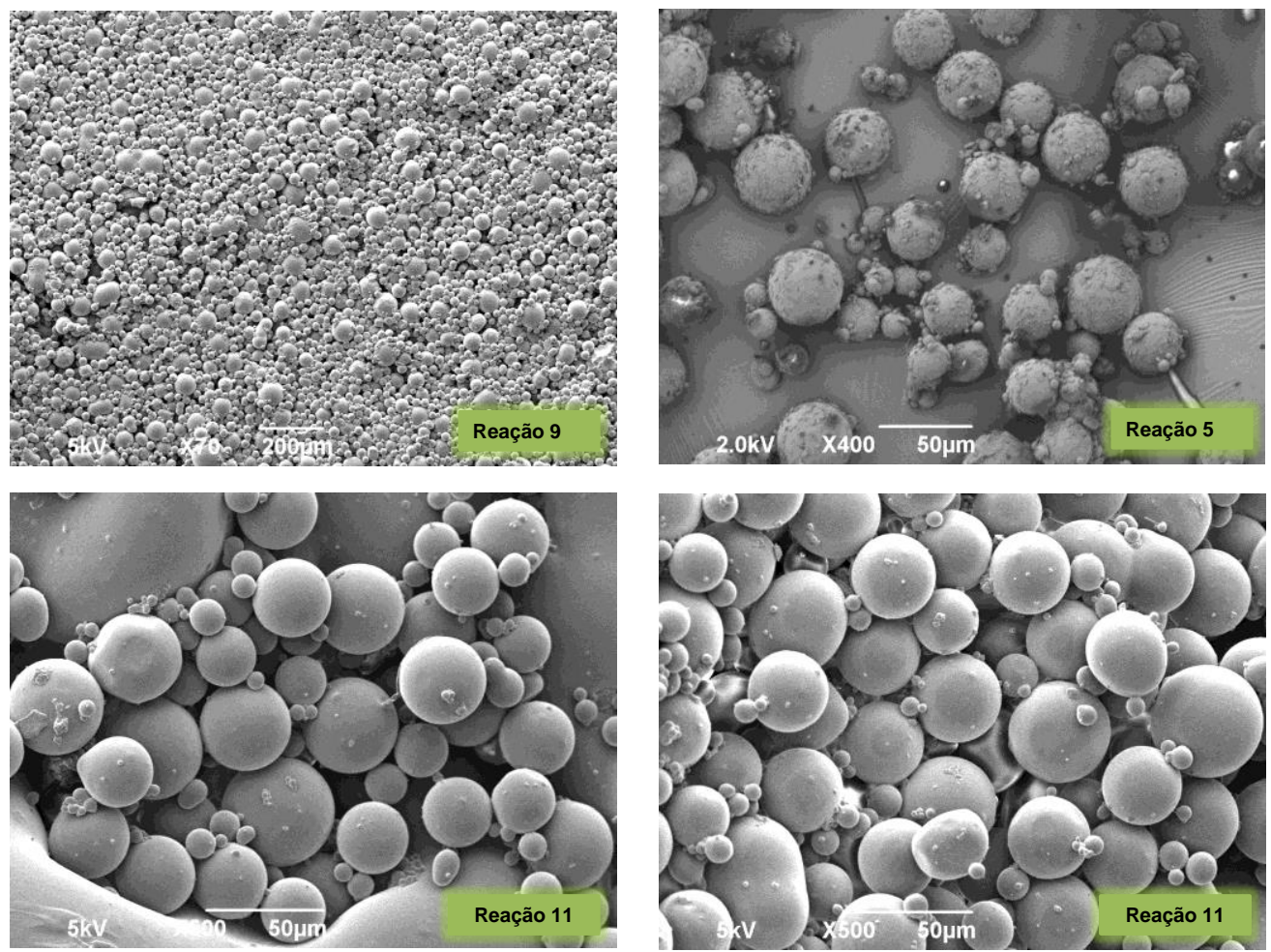

Figura 2 - Micrografias (MEV) do polímero PMMA obtido por polimerização em $\mathrm{CO}_{2}$ supercrítico. 
Com o objetivo de verificar a reatividade do agente estabilizante utilizado e, também, a eficiência do processo em $\mathrm{CO}_{2}$ denso para a obtenção de PMMA, foram conduzidas análises de $\mathrm{RMN}-\mathrm{H}^{+}$nos polímeros, como é mostrado na Figura 3 para a reação 1. Tendo em vista que os parâmetros operacionais que interferem na cinética de reação não foram alterados, é mostrada apenas a análise para uma das reações. Os resultados obtidos para as amostras analisadas demonstram que a molécula formada no processo foi a do PMMA, sem a ocorrência de reações secundárias relevantes entre o agente estabilizante e os radicais do iniciador ou cadeias menores de polímero em crescimento. Esse resultado é importante na determinação da adequação do PDMS-vinil terminal para o processo, não interferindo na cinética de polimerização do MMA em condições supercríticas.

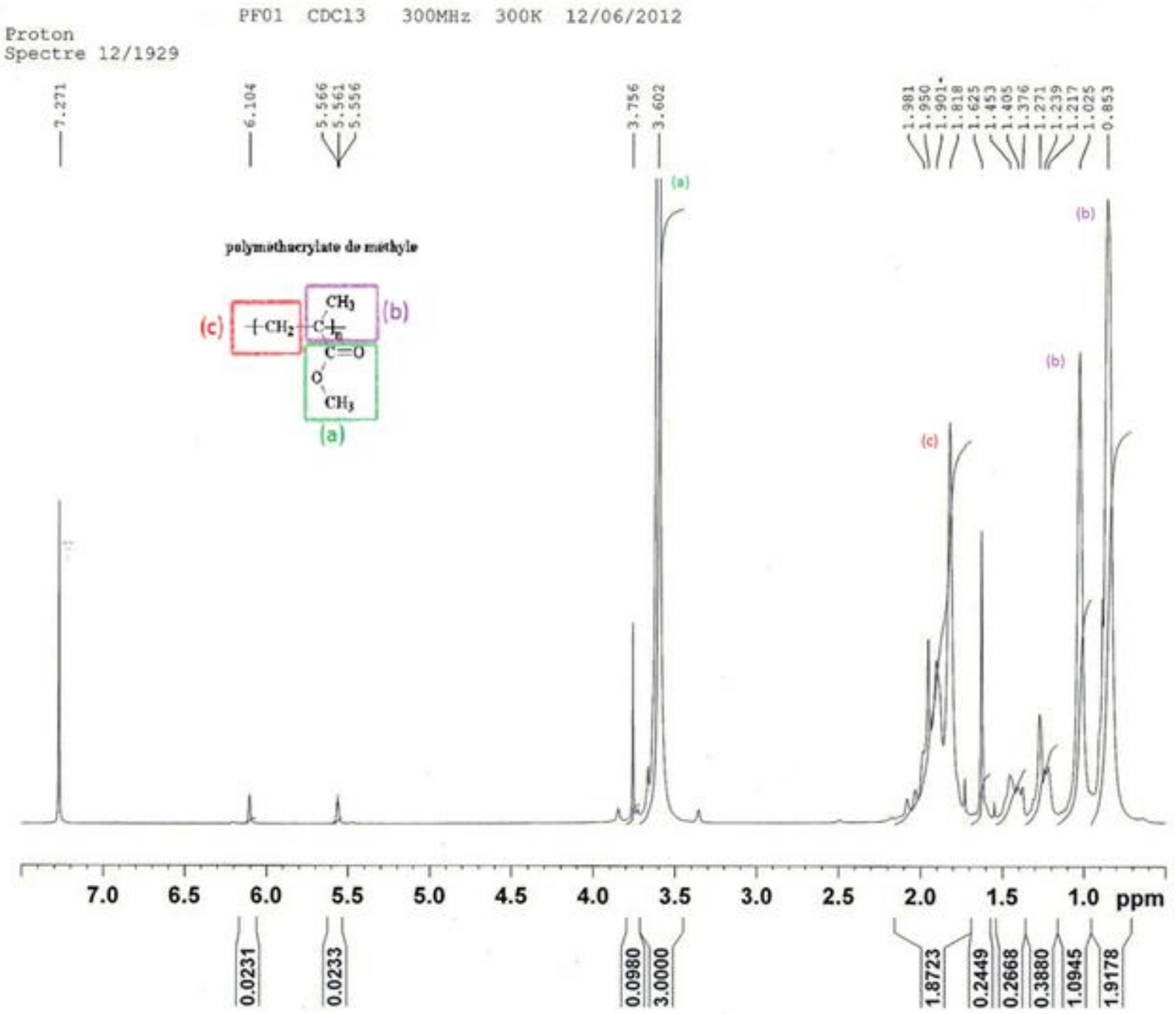

Figura 3 - Análise de $\mathrm{RMN}-\mathrm{H}^{+}$para a reação 1. 


\section{CONCLUSÕES}

Nesse trabalho foi possível obter poli(metracrilato de metila) por dispersão em $\mathrm{CO}_{2}$ supercrítico, utilizando o agente dispersante PDMS-vinil terminal. Estabilizantes à base de compostos fluorados e siloxanos são utilizados em sistemas de polimerização em condições supercríticas devido à afinidade pelo $\mathrm{CO}_{2}$ e pelos monômeros mais utilizados nesse sistema (estireno e metacrilato de metila). $\mathrm{O}$ agente estabilizante definido nesse trabalho se mostrou adequado nas condições avaliadas, garantindo a estabilidade da reação, a qualidade do produto final e a um custo menor do que se utilizado um estabilizante sintético ou fluorado. Não se tem relatos na literatura até o momento do uso do PDMS-vinil terminal para essa finalidade. A condição de $16 \mathrm{MPa}$ e $80^{\circ} \mathrm{C}$ se mostrou apropriada para a polimerização do MMA em $\mathrm{CO}_{2}$ supercrítico. Dessa forma, o sistema reacional proposto se mostrou eficiente para o objetivo do trabalho. A avaliação mais aprofundada dos parâmetros é necessária para melhor conhecimento e caracterização do produto e do meio reacional.

\section{REFERÊNCIAS}

BEUERMANN, S.; BUBACK, M. Supercritical Carbon Dioxide in Polymer Reaction Engineering, Wiley-VCH, 2005.

De SIMONE, J. M.; MAURY, E. E.; MENCELOGLU, Y. Z.; McClAIN, J. B.; ROMACK, T. J.; COMBES, J. R. Science 1994, 265, 356.

HAN, Sang-Hun; PARK, Kyung-Kyu; LEE, Sang-Ho. Polymerization of methyl methacrylate in carbon dioxide using glycidyl methacrylate linked reactive stabilizer: Effect of pressure, reaction time, and mixing. Macromolecular Research, 17, 51-57, 2009.

LACROIX-DESMAZES, P. "Polymérisations en milieu fluide supercritique", in Les latex synthétiques: élaboration et applications, Tec\&Doc-Lavoisier, Paris, J.-C. Daniel, Ch. Pichot (Eds.), [ISBN: 2-7430-0820-2], (2006), 33, 911-933.

PARK, Jin-Yeol; SHIM, Jae-Jin. Emulsion stability of PMMA particles formed by dispersion polymerization of methyl methacrylate in supercritical carbon dioxide, J. of Supercritical Fluids, 27, 297-307, 2003.

REVERCHON, E.; ADAMI, R.; CARDEA, S.; DELLA PORTA, G. Supercritical fluids processing of polymers for pharmaceutical and medical applications. Review. Journal of Supercritical Fluids, 47, 484-492, 2009. 